ARTÍCULO ORIGINAL

\title{
Adherencia al tratamiento farmacológico y control glucémico en pacientes adultos con diabetes mellitus tipo 2
}

Guillermo Guzmán ${ }^{1}$, Andrea Arce², Harold Saavedra², Mayra Rojas², Juan Sebastián Solarte², Miguel Mina², Kenny Gutiérrez ${ }^{3}$ y Veline Martínez ${ }^{4^{*}}$

${ }^{1}$ Departamento de Endocrinología, Universidad ICESI, Fundación Valle del Lili; ${ }^{2}$ Facultad de medicina, pregrado, Universidad ICESI; ${ }^{3}$ Departamento de Endocrinología, Fundación Valle del Lili; ${ }^{4}$ Departamento de Medicina Interna, Universidad ICESI, Fundación Valle del Lili. Cali, Colombia

\section{RESUMEN}

Introducción: La diabetes mellitus tipo 2 (DMT2) es una enfermedad crónica con alta prevalencia. Requiere un tratamiento eficaz para lograr el control glucémico y así reducir el impacto de la enfermedad en la salud del paciente y los costos. La adherencia al tratamiento es de vital importancia para el logro de estos objetivos. Este estudio buscó determinar la adherencia al tratamiento farmacológico y su relación con el control glucémico. Materiales y métodos: Estudio descriptivo y transversal que incluye pacientes mayores de 18 años, con diagnóstico de DMT2, en quienes se aplicó la escala de Morinsky Green para medir adherencia, con el objetivo de determinar la relación entre esta y el control glucémico. Resultados: De 201 pacientes, el $61.7 \%$ eran mujeres. La edad promedio fue de 63.1 años. La prevalencia de la adherencia al tratamiento farmacológico fue del $47.8 \%$. El $53.7 \%$ de los pacientes pre-

\section{ABSTRACT}

Introduction: Type 2 diabetes mellitus (DMT2) is a chronic disease with high prevalence. It requires effective management to achieve control goals and thus reduce impact on patient health and costs. Adherence to treatment is key in this process. This study aims to determine adherence to pharmacological treatment and its relation to glycemic control. Materials and methods: Descriptive, cross-sectional study. It includes patients over 18 years of age, diagnosed with DMT2, in whom the Morinsky green scale was applied to measure adherence, in order to determine the relationship between glycemic control and adherence. Results: Of 201 patients, $61.7 \%$ were women. The average age was 63.1 years. The prevalence of adherence to pharmacological management was $47.8 \%$. Adequate glycemic control was observed in $53.7 \%$. Women and black people were more adherents $(p<0.05)$. The relation- 
sentaron un control glucémico adecuado. Se observó mayor adherencia entre mujeres y personas con nivel académico más alto. La relación entre adherencia y control no fue estadísticamente significativa ( $p=0.310)$. Conclusiones: No hubo relación estadísticamente significativa entre control glucémico y adherencia. Es necesario realizar otros estudios para aclarar qué otros factores pueden estar influyendo en el control glucémico. Se recomienda revaluar la forma de medir adherencia en nuestra población, de tal forma que prediga el control glucémico.

Palabras clave: Adherencia. Control glucémico. HbA1c. Prevalencia. ship between adherence and control was not statistically significant $(p=0.310)$. Conclusions: Relationship between glycemic control and adherence was not statistically significant. Studies should be conducted to clarify which other factors may be influencing glycemic control. It is recommended to re-evaluate the measurement of adherence in our population in such a way it can be used to predict glycemic control. (Rev ALAD. 2018;8:35-43) Corresponding author: Veline Martínez, velinemartinez@gmail.com

Key words: Adherence. Glycemic control. HbA1c. Prevalence.

\section{INTRODUCCIÓN}

La diabetes mellitus (DM) es una enfermedad con un fuerte impacto en la salud pública, debido a su alta frecuencia, las complicaciones asociadas y los costos que implica para las naciones. En el 2013, 347 millones de personas en el mundo padecían DM¹. En Colombia, la prevalencia de DMT2 era del 7,3\% en 2013, con una tasa de mortalidad anual estimada en 2014 de 14.03/100.000 habitantes². Un mal control glucémico puede llevar a complicaciones macro y microvasculares que deterioran la calidad de vida de los pacientes y aumentan los costos en salud ${ }^{3,4}$.

Un tratamiento eficaz puede mejorar los desenlaces relacionados con complicaciones de la DM. Mantener cifras de glucemia cercanas a los valores normales, expresado en términos de hemoglobina glucosilada (HbA1c) con un valor menor del $7 \%$, permite disminuir el impacto de la enfermedad ${ }^{5-7}$. La adherencia al tratamiento por parte del paciente es importante, lo que se ve reflejado en disminución de la HbA1c, mortalidad, tasas de hospitalización y, por ende, menores gastos en salud. La tasa de adherencia al tratamiento en la DM es variable según el instrumento de medición y puede oscilar entre el 39 y el $93 \%{ }^{8}$.

Con este estudio se buscó establecer la prevalencia de la adherencia al tratamiento farmacológico y su relación con el control glucémico, en pacientes con DMT2 tratados en la Fundación Valle del Lili, centro de alto nivel de complejidad y referencia nacional, así como explorar los factores relacionados con la adherencia y control glucémico.

\section{MATERIALES Y MÉTODOS}

Se llevó a cabo un estudio epidemiológico observacional, descriptivo y transversal. La población correspondió a pacientes adultos con diagnóstico de DMT2 que acuden a consulta externa de 
endocrinología y medicina interna de la Fundación Valle del Lili, institución de alta complejidad. Fueron elegibles quienes tenían diagnóstico confirmado de DMT2 (código ClE-10 de E10-E149), según los registros de asistentes a la consulta, en el periodo comprendido entre el 1 de septiembre de 2016 y el 30 de abril de 2017. Se incluyeron los mayores de 18 años que hubieran tenido control dentro de los tres meses anteriores, con informe de HbA1c y que fueran capaces de contestar el cuestionario para medir la adherencia, antes de cuya administración se firmó el consentimiento informado.

Se realizó un cálculo de tamaño de muestra según la población atendida en el año 2016, según una prevalencia de adherencia reportada en la literatura del 79.3\% (de acuerdo al estudio realizado en Bogotá, Colombia, dada la similitud de la población a estudiar), con un nivel de confianza del 95\%, una precisión del $5 \%$ y un resultado de 226 pacientes en total. Se realizó un muestreo aleatorio simple y se elaboró una base de datos para el registro electrónico en BDClinic. Se incluyeron variables demográficas como edad, sexo y tipo de afiliación al sistema de salud, teniendo en cuenta que en Colombia el acceso a los servicios de salud está relacionado con esta. El régimen de afiliación se definió así: contributivo (afiliados con capacidad de pago para acceder al servicio de salud), subsidiado (población pobre, sin capacidad de pago, que accede a los servicios de salud por subsidios del Estado), medicina prepagada (afiliados a planes adicionales de salud), régimen especial (afiliados a programas excepcionales en salud, que tienen un sistema propio de cobertura). También se registraron nivel académico y el estrato socioeconómico. Considerado este como una aproximación a la diferencia demográfica jerarquizada, se basa en las condiciones de la vivienda y aspectos poblacionales. Los estratos 1, 2 y 3 corresponden a estratos bajos; se trata de usuarios con menores recursos, que son beneficiarios de subsidios. Los estratos 5 y 6 , altos, engloban a usuarios con mayores recursos económicos, que deben pagar los sobrecostos. El estrato 4 no es beneficiario de subsidios ni debe pagar sobrecostos. Así mismo, se incluyeron la situación de convivencia, la procedencia, la presencia de comorbilidades y el tabaquismo, y se tomaron datos de hospitalizaciones recientes. También se registraron variables relacionadas con el tiempo de evolución de la enfermedad, el tipo de tratamiento recibido, el número de tabletas que el paciente tomaba y estudios de laboratorio (en este caso, la HbA1c).

Para medir la adherencia farmacológica, se utilizó la escala de Morinsky Green y se definió como adherente al paciente que respondía de forma correcta (No/Sí/No/No) al siguiente cuestionario:

- ¿Olvida alguna vez tomar los medicamentos para tratar su enfermedad?

- ¿Toma los medicamentos a las horas indicadas?

- Cuando se encuentra bien, ¿deja de tomar la medicación?

- Si alguna vez se siente mal, ¿deja usted de tomarla?

Para evaluar el control metabólico se tomó como referencia el valor de HbA1c con la que acudía el paciente al control y este se consideró controlado si tenía un valor del $7 \%$ o menor.

Para el análisis estadístico, las variables cuantitativas se describieron mediante medias o medianas y medidas de dispersión, desviación estándar y rango intercuartílico, de acuerdo con el cumplimiento de supuestos de normalidad tras la prueba de Kolmogórov-Smirnov. Las variables categóricas se describieron como el valor absoluto y el porcentaje. Se realizaron tablas de frecuencia de acuerdo con la 
presencia y ausencia de adherencia y control glucémico. Se compararon adherentes o no, controlados o no, con $\chi^{2}$ o test exacto de Fisher para las variables cualitativas y con la prueba de t de Student o Wilcoxon Mann-Whitney en las cuantitativas, según el cumplimiento de supuestos, con grado de significación del 0.05. Para evaluar la asociación de factores determinantes se realizó un modelo de regresión logística con selección de variables stepwise-backward, se comienza por considerar incluidas en el modelo teórico a todas las variables disponibles y se van eliminando de este de una en una según su capacidad explicativa . El análisis estadístico se realizó con STATA13. El presente estudio tuvo aprobación del comité de ética en investigación biomédica institucional.

\section{RESULTADOS}

De un total de 1,982 pacientes registrados en la Fundación Valle del Lili con DMT2 en 2016, se seleccionaron por muestreo aleatorizado simple 226 y se descartaron 25 por datos incompletos en la historia clínica, quedando un total de 201 sujetos para el análisis (Fig. 1). La prevalencia de la adherencia al tratamiento farmacológico de la diabetes fue del 47.8\% (96 de 201 pacientes).

Las características de la población están representadas en la tabla 1. El promedio de edad fue de 63.1 años, el 61.7\% eran mujeres, el $94.03 \%$ procedían del área urbana y el $58.71 \%$ pertenecían al régimen contributivo. Se observó hipertensión arterial en el $60.7 \%$, dislipidemia en el $40.8 \%$, enfermedad coronaria en el $15.9 \%$ y enfermedad renal crónica (ERC) en el $15 \%$; el $23.4 \%$ de los participantes fumaba (Tabla 2 ). En el $43.78 \%$ de los participantes, el diagnóstico de DM se había realizado hacía 10 o más años; el 40.3\% recibía tratamiento con antidiabéticos orales y el $25.87 \%$, terapia oral más insulina (Tabla 3). La ERC estadio 4 y el recibir mayor número de tabletas para tratamiento (por diversas comorbilidades) estuvieron relacionados con no adherencia. Se relacionaron

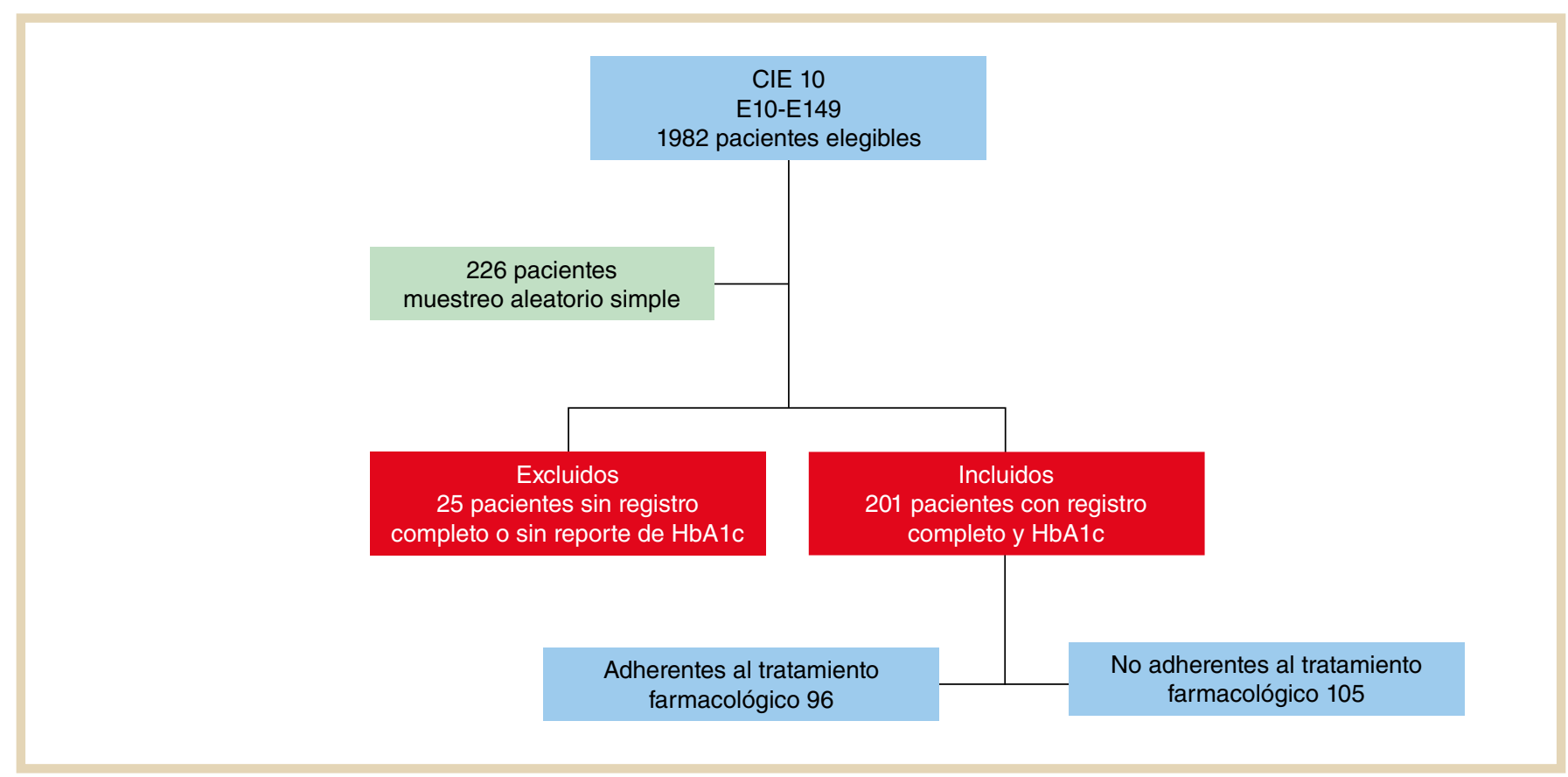

Figura 1. Flujograma de pacientes en el estudio. 
TABLA 1. Características generales de los pacientes con DMT2 en relación a la adherencia y el control glucémico

\begin{tabular}{|c|c|c|c|c|c|c|c|c|c|}
\hline \multirow[t]{2}{*}{ Característica } & \multirow{2}{*}{$\begin{array}{c}\text { Total } \\
\text { pacientes } \\
\mathbf{n}=\mathbf{2 0 1}\end{array}$} & \multicolumn{2}{|c|}{ Adherencia } & \multirow[t]{2}{*}{$\mathbf{p}$} & \multirow[t]{2}{*}{ OR } & \multicolumn{2}{|c|}{ Control } & \multirow[t]{2}{*}{$\mathrm{p}$} & \multirow[t]{2}{*}{ OR } \\
\hline & & $\begin{array}{c}\text { Sí } \\
96(47.8)\end{array}$ & $\begin{array}{c}\text { No } \\
105(52.2)\end{array}$ & & & $\begin{array}{c}\text { Sí } \\
108(53.7)\end{array}$ & $\begin{array}{c}\text { No } \\
93(46.3)\end{array}$ & & \\
\hline Edad** & $63.1 \pm 12$ & 62 & 63 & 0.1987 & & 63 & 61 & 0.282 & \\
\hline $\begin{array}{l}\leq 40 \text { años } \\
41-60 \text { años } \\
61-80 \text { años } \\
>80 \text { años }\end{array}$ & $\begin{array}{r}8 \\
74 \\
100 \\
19\end{array}$ & $\begin{array}{c}5(62.5) \\
37(50.0) \\
48(48.0) \\
6(31.58)\end{array}$ & $\begin{array}{l}3(37.5) \\
37(50.0) \\
52(52.0) \\
13(68.42)\end{array}$ & & $\begin{array}{c}1 \\
1.66(0.37-7.48) \\
1.80(0.41-7.96) \\
3.61(0.64-20.32)\end{array}$ & $\begin{array}{l}3(37.5) \\
37(50.0) \\
58(58.0) \\
10(52.63)\end{array}$ & $\begin{array}{c}5(62.5) \\
37(50.0) \\
42(42.0) \\
9(47.37)\end{array}$ & & $\begin{array}{c}1 \\
0.36(0.71-1.91) \\
0.20(0.38-1.09) \\
0.30(0.04-2.18)\end{array}$ \\
\hline Sexo & & & & 0.093 & & & & 0.689 & \\
\hline Hombres & 77 & $31(40.26)$ & $46(59.74)$ & & 1 & $40(51.95)$ & $37(48.85)$ & & 1 \\
\hline Mujeres & 124 & $65(52.42)$ & $59(47.58)$ & & $0.79(0.39-1.62)$ & $68(54.84)$ & $56(45.16)$ & & $0.43(0.20-0.90)$ \\
\hline $\begin{array}{l}\text { Nivel } \\
\text { académico }\end{array}$ & & & & $0.049^{*}$ & & & & 0.086 & \\
\hline Analfabeto & 2 & $1(50)$ & $1(50)$ & & 1 & $0(0.00)$ & $2(100)$ & & 1 \\
\hline Primaria & 79 & $41(51.90)$ & $38(48.10)$ & & $1.29(0.05-12.82)$ & $38(48.10)$ & $41(51.9)$ & & $2.61(0.95-7.14)$ \\
\hline Secundaria & 48 & $26(54.17)$ & $22(45.83)$ & & $0.47(0.22-0.92)^{*}$ & $23(47.92)$ & $25(52.08)$ & & $1.81(0.71-4.61)$ \\
\hline Técnico & 17 & $11(64.70)$ & $6(35.30)$ & & $0.42(0.18-0.92)^{*}$ & $12(70.59)$ & $5(29.41)$ & & $0.49(0.13-1.88)$ \\
\hline Profesional & 55 & $17(30.9)$ & $38(69.1)$ & & $0.31(0.94-0.97) *$ & $35(63.64)$ & $20(36.36)$ & & $0.58(0.44-2.01)$ \\
\hline $\begin{array}{l}\text { Régimen } \\
\text { seguridad } \\
\text { social }\end{array}$ & & & & 0.123 & & & & 0.284 & \\
\hline Prepagada & 77 & $33(42.86)$ & $44(57.14)$ & & 1 & $46(59.74)$ & $31(40.26)$ & & 1 \\
\hline Contributivo & 118 & $62(52.54)$ & $56(47.46)$ & & $2.59(0.83-5.22)$ & $60(50.85)$ & $58(49.15)$ & & $0.94(0.16-4.40)$ \\
\hline Particular & 6 & $1(16.67)$ & $5(83.33)$ & & $1.9(0.26-12.85)$ & $2(33.33)$ & $4(66.67)$ & & $0.85(0.78-1.89)$ \\
\hline Subsidiado & 0 & & & & - & & & & - \\
\hline $\begin{array}{l}\text { Situación de } \\
\text { convivencia }\end{array}$ & & & & 0.466 & & & & 0.398 & \\
\hline Vive solo & 14 & $8(57.14)$ & $6(42.86)$ & & 1 & $6(42.86)$ & $8(57.14)$ & & 1 \\
\hline $\begin{array}{l}\text { Vive } \\
\text { acompañado }\end{array}$ & 187 & $88(47.06)$ & 99 (52.94) & & $0.89(0.87-5.23)$ & $102(54.55)$ & $85(45.45)$ & & $0.58(0.15-2.17)$ \\
\hline Procedencia & & & & $0.015^{*}$ & & & & 0.387 & \\
\hline Rural & 12 & $10(83.33)$ & $2(16.67)$ & & 1 & $5(41.67)$ & $7(58.33)$ & & 1 \\
\hline Urbana & 189 & $86(45.50)$ & $103(54.50)$ & & $0.2(0.04-0.97)^{*}$ & $103(54.5)$ & $86(45.50)$ & & $0.99(0.23-4.18)$ \\
\hline $\begin{array}{l}\text { Estrato } \\
\text { socioeconó- } \\
\text { mico }\end{array}$ & & & & 0.087 & & & & 0.143 & \\
\hline 1 & 7 & $6(85.71)$ & $1(14.29)$ & & 1 & $1(14.29)$ & $6(85.71)$ & & 1 \\
\hline 2 & 41 & $22(53.66)$ & $19(46.34)$ & & $1.91(0.48-7.66)$ & $17(41.46)$ & $24(58.54)$ & & $0.43(0.11-1.61)$ \\
\hline 3 & 64 & $34(53.12)$ & $30(46.88)$ & & $1.34(0.35-5.18)$ & $36(56.25)$ & $28(43.75)$ & & $0.32(0.89-1.14)$ \\
\hline
\end{tabular}


TABLA 1. Características generales de los pacientes con DMT2 en relación a la adherencia y el control glucémico (Continuación)

\begin{tabular}{|c|c|c|c|c|c|c|c|c|c|}
\hline \multirow[t]{2}{*}{ Característica } & \multirow{2}{*}{$\begin{array}{c}\text { Total } \\
\text { pacientes } \\
\text { n = 201 }\end{array}$} & \multicolumn{2}{|c|}{ Adherencia } & \multirow[t]{2}{*}{ p } & \multirow[t]{2}{*}{ OR } & \multicolumn{2}{|c|}{ Control } & \multirow[t]{2}{*}{ p } & \multirow[t]{2}{*}{ OR } \\
\hline & & $\begin{array}{c}\text { Sí } \\
96(47.8)\end{array}$ & $\begin{array}{c}\text { No } \\
105(52.2)\end{array}$ & & & $\begin{array}{c}\text { Sí } \\
108 \text { (53.7) }\end{array}$ & $\begin{array}{c}\text { No } \\
93(46.3)\end{array}$ & & \\
\hline 4 & 32 & $11(34.38)$ & $21(65.62)$ & & $2.52(0.54-11.62)$ & $21(65.62)$ & $11(34.38)$ & & $0.16(0.36-0.72)$ \\
\hline 5 & 33 & $15(45.45)$ & $18(54.55)$ & & $1.2(0.25-5.66)$ & $19(57.58)$ & $14(42.42)$ & & $0.41(0.98-1.78)$ \\
\hline 6 & 16 & $4(25)$ & $12(75)$ & & $2.5(0.37-17.04)$ & $10(62.0)$ & $6(37.5)$ & & $0.3(0.53-1.67)$ \\
\hline No disponible & 8 & $4(50)$ & $4(50)$ & & - & $4(50)$ & $4(50)$ & & - \\
\hline Estado civil & & & & 0.691 & & & & 0.531 & \\
\hline Soltero & 29 & $17(58.62)$ & $12(41.38)$ & & 1 & $14(48.28)$ & $15(51.72)$ & & 1 \\
\hline Separado & 17 & $9(52.94)$ & $8(47.06)$ & & $2.09(0.83-5.22)$ & $7(41.18)$ & $10(58.82)$ & & 2.84 \\
\hline Casado & 137 & $62(45.25)$ & $75(54.75)$ & & $1.83(0.26-12.85)$ & $75(54.74)$ & $62(42.25)$ & & 2.4 \\
\hline Viudo & 18 & $8(44.44)$ & $10(55.56)$ & & $1.9(0.47-7.92)$ & $12(66.67)$ & $6(33.33)$ & & 1.77 \\
\hline
\end{tabular}

Valores expresados como valor absoluto (porcentaje).

${ }^{*} \mathrm{p}<0.05$

**Reportado como media (desviación estándar).

DMT2: diabetes mellitus tipo 2; OR: odds ratio (intervalo de confianza).

TABLA 2. Comorbilidades asociadas a la DMT2 y su relación con la adherencia y el control glucémico

\begin{tabular}{|c|c|c|c|c|c|c|c|c|c|}
\hline \multirow[t]{2}{*}{ Patología } & \multirow{2}{*}{$\begin{array}{c}\text { Total } \\
\text { pacientes } \\
\mathbf{n}=\mathbf{2 0 1}\end{array}$} & \multicolumn{2}{|c|}{ Adherencia } & \multirow[t]{2}{*}{ p } & \multirow[t]{2}{*}{ OR } & \multicolumn{2}{|c|}{ Control } & \multirow[t]{2}{*}{ p } & \multirow[t]{2}{*}{ OR } \\
\hline & & $\begin{array}{c}\text { Sí } \\
96(47.76)\end{array}$ & $\begin{array}{c}\text { No } \\
105(52.24)\end{array}$ & & & $\begin{array}{c}\text { Sí } \\
108(53.73)\end{array}$ & $\begin{array}{c}\text { No } \\
93(46.27)\end{array}$ & & \\
\hline HTA & $122(60.7)$ & $56(45.9)$ & $66(54.10)$ & 0.512 & $1.02(0.48-2.18)$ & $63(51.64)$ & $59(48.36)$ & 0.46 & $1.26(0.69-2.30)$ \\
\hline Dislipidemia & $82(40.8)$ & $35(42.68)$ & $47(57 \cdot 32)$ & 0.232 & $1.48(0.73-3.01)$ & $47(57.32)$ & $35(42.68)$ & 0.397 & $0.81(0.44-1.46)$ \\
\hline ECV & $5(2.5)$ & $2(40.0)$ & $3(60.0)$ & 0.542 & $1.48(0.07-20.44)$ & $4(80.0)$ & $1(20.0)$ & 0.375 & $0.32(0.31-3.28)$ \\
\hline Enf. coronaria & $32(15.9)$ & $14(43.75)$ & $18(56.25)$ & 0.620 & $0,95(0.35-2.58)$ & $21(65.62)$ & $11(34.38)$ & 0.233 & $0.59(0.24-1.39)$ \\
\hline ERC & & & & $0.009^{*}$ & & & & 0.551 & \\
\hline ERC 1-3 (a-b) & 26 (12.9) & $6(23.08)$ & $20(76.92)$ & & $0.75(0.05-10.6)$ & $12(46.15)$ & $14(53.85)$ & & 1 \\
\hline ERC 4 & $1(0.5)$ & $0(0.0)$ & $1(100)$ & & $4.3(1.56-11.85)^{*}$ & $1(100.00)$ & $0(0.0)$ & & $0.42(0.03-5.1)$ \\
\hline ERC 5 o diálisis & $3(1.6)$ & $1(33 \cdot 33)$ & $2(66.67)$ & & $8.1(0.44-10.83)$ & $1(33 \cdot 33)$ & $2(66.67)$ & & $0.63(0.04-8.3)$ \\
\hline Neoplasia & $24(11.9)$ & $13(54.17)$ & $11(45.83)$ & 0.503 & $0.8(0.28-2.44)$ & $14(58.33)$ & $10(41.67)$ & 0.630 & $0.75(0.3-1.84)$ \\
\hline EPOC & $8(3.9)$ & $3(37.5)$ & $5(62.5)$ & 0.723 & $1.2(0.18-2.44)$ & $5(62.5)$ & $3(37.5)$ & 0.727 & $1.5(0.27-8.22)$ \\
\hline $\begin{array}{l}\text { Insuficiencia } \\
\text { cardíaca }\end{array}$ & $7(3.48)$ & $2(28.57)$ & $5(71.43)$ & 0.448 & $7.5(0.33-17.1)$ & $5(71.43)$ & $2(28.57)$ & 0.454 & $0.36(0.06-2.27)$ \\
\hline Cirrosis & $10(4.9)$ & $4(40.0)$ & $6(60.0)$ & 0.750 & $1.2(0.27-6.01)$ & $8(80.0)$ & $2(20.0)$ & 0.110 & $0.21(0.04-1.06)$ \\
\hline Tabaquismo & $41(20.4)$ & $20(48.78)$ & $21(51.22)$ & 0.884 & $0.27(0.3-1.7)$ & $27(65.85)$ & $14(34.15)$ & 0.081 & $0.65(0.28-1.31)$ \\
\hline $\begin{array}{l}\text { Hospitalización } \\
\text { reciente }\end{array}$ & $12(5.9)$ & $8(66.67)$ & $4(33.33)$ & 0.236 & $0.16(0.26-1.03)$ & $7(58.33)$ & $5(41.67)$ & 0.775 & $0.98(0.27-3.51)$ \\
\hline
\end{tabular}

Valores expresados como valor absoluto (porcentaje).

*p $<0.05$

DMT2: diabetes mellitus tipo 2; HTA: hipertensión arterial; ECV: enfermedad cerebrovascular; ERC: enfermedad renal crónica; EPOC: enfermedad pulmonar obstructiva crónica; ICC: Insuficiencia cardiaca crónica; OR: odds ratio (intervalo de confianza). 
TABLA 3. Tiempo de evolución de la DMT2, tratamiento de la DMT2 y relación con la adherencia y el control glucémico

\begin{tabular}{|c|c|c|c|c|c|c|c|c|c|}
\hline \multirow[t]{2}{*}{ Característica } & \multirow{2}{*}{$\begin{array}{c}\text { Total } \\
\text { pacientes } \\
\text { n = 201 }\end{array}$} & \multicolumn{2}{|c|}{ Adherencia } & \multirow[t]{2}{*}{$\mathrm{p}$} & \multirow[t]{2}{*}{ OR } & \multicolumn{2}{|c|}{ Control } & \multirow[t]{2}{*}{$\mathbf{p}$} & \multirow[t]{2}{*}{ OR } \\
\hline & & $\begin{array}{c}\text { Sí } \\
96(47 \cdot 76)\end{array}$ & $\begin{array}{c}\text { No } \\
105(52.24)\end{array}$ & & & $\begin{array}{c}\text { Sí } \\
108(53.73)\end{array}$ & $\begin{array}{c}\text { No } \\
93(46.27)\end{array}$ & & \\
\hline $\begin{array}{l}\text { Duración DMT2 } \\
\text { (meses) }\end{array}$ & & & & 0.463 & & & & 0.006 & \\
\hline $0-12$ & 15 & $5(33.33)$ & $10(66.67)$ & & 1 & $10(66.67)$ & $5(33 \cdot 33)$ & & 1 \\
\hline $13-60$ & 45 & $25(55.56)$ & $20(44.44)$ & & $0.4(0.11-1.42)$ & $28(62.22)$ & $17(37.78)$ & & $0.9(0.25-3.38)$ \\
\hline $61-120$ & 53 & $26(49.06)$ & $27(50.94)$ & & $0.6(0.1-2.01)$ & $35(66.04)$ & $18(33.96)$ & & $0.8(0.22-2.89)$ \\
\hline$>120$ & 88 & $40(45.45)$ & $48(54.55)$ & & $0.6(0.18-1.92)$ & $35(39.77)$ & $53(60.23)$ & & $2.1(1.63-7.22)$ \\
\hline \multicolumn{10}{|l|}{$\begin{array}{l}\text { Tratamiento } \\
\text { DMT2 }\end{array}$} \\
\hline Solo oral & 81 & $41(50.62)$ & $40(49.38)$ & 0.626 & 1 & $56(69.14)$ & $25(30.86)$ & $0.016 *$ & 1 \\
\hline Solo GLP1 & 2 & $2(100.00)$ & $0(0.0)$ & & 1 & $1(50.0)$ & $1(50.0)$ & & $2.4(0.14-42.84)$ \\
\hline $\mathrm{GLP} 1+$ oral & 5 & $3(85.71)$ & $2(14.29)$ & & $0.8(0.18-5.1)$ & $2(14.29)$ & $3(85.71)$ & & $4.1(0.60-28.34)$ \\
\hline Solo insulina & 32 & $17(53.12)$ & $15(46.88)$ & & $0.8(0.3-1.8)$ & $12(37.5)$ & $20(62.5)$ & & $3.3(1.35-7.96)$ \\
\hline Insulina + oral & 52 & $21(40.38)$ & $31(59.52)$ & & $1.5(0.72-3.1)$ & $21(40.38)$ & $31(59.52)$ & & $2.7(1.22-5.76)^{*}$ \\
\hline Insulina + GLP1 & 16 & $7(43.75)$ & $9(56.25)$ & & $1.5(0.29-4.5)$ & $8(50.0)$ & $8(50.0)$ & & $1.9(0.60-5.80)$ \\
\hline $\begin{array}{l}\text { Insulina + GLP1 } \\
\text { + oral }\end{array}$ & 13 & $5(38.46)$ & $8(61.54)$ & & $1.5(0.5-3.2)$ & $\begin{array}{c}8(61.54) 5 \\
(38.46)\end{array}$ & & & $1.2(0.33-4.28)$ \\
\hline $\begin{array}{l}\text { Número } \\
\text { tabletas }\end{array}$ & & & & 0.019 & & & & 0.712 & \\
\hline $0-4$ & 50 & $32(64.0)$ & $18(36.0)$ & & 1 & $\begin{array}{c}29(58.0) 21 \\
(42.0)\end{array}$ & & & 1 \\
\hline $5-10$ & 117 & $52(44.44)$ & $65(55.56)$ & & $1.7(0.95-3.2)$ & $\begin{array}{c}60(51.28) 57 \\
(48.72)\end{array}$ & & & $0.7(0.37-1.36)$ \\
\hline$>10$ & 34 & $12(35.29)$ & $22(64.71)$ & & $6.6(0.7-62)$ & $\begin{array}{c}19(55.88) 15 \\
(44.12)\end{array}$ & & & $3.3(0.46-23.26)$ \\
\hline
\end{tabular}

significativamente con la adherencia el nivel académico y la procedencia de área urbana, comportándose estos como factores que favorecían la adherencia.

En cuanto al control glucémico, estaban controlados $(\mathrm{HbA} 1 \mathrm{c} \leq 7)$ el $53.7 \%$, el $9.3 \%$ tenían $\mathrm{HbA} 1 \mathrm{c}>9 \%$ (Tabla 4) y ninguna patología de base se asoció con estar o no controlado (Tabla 2). El tiempo de duración de la DMT2 y el tipo de tratamiento mostraron asociación significativa con el control glucémico: el mayor tiempo de duración de la enfermedad aumenta el riesgo de no control, lo mismo que el recibir terapia combinada (insulina con medicación oral). La relación entre adherencia al manejo farmacológico y control glucémico no fue significativa $(p=0.310)$. 
TABLA 4. Hemoglobina glucosilada en relación a tiempo de evolución de diabetes, tratamiento de la enfermedad y adherencia

\begin{tabular}{|c|c|c|c|c|c|c|c|c|}
\hline \multirow[t]{2}{*}{$\mathrm{HbA}_{1 \mathrm{C}}$} & \multicolumn{2}{|c|}{ Tiempo duración DM* } & \multicolumn{4}{|c|}{ Manejo DM* } & \multicolumn{2}{|c|}{ Adherencia } \\
\hline & $\leq 5$ años & $>5$ años & Oral & Insulina & GLP1 & Combinados & Sí & No \\
\hline & $\%$ & $\%$ & $\%$ & $\%$ & $\%$ & $\%$ & $\%$ & $\%$ \\
\hline$\leq 7$ & 18.9 & 34.8 & 27.9 & 5.7 & 0.5 & 19.4 & 23.9 & 29.8 \\
\hline $7.01-8.0$ & 6.9 & 20.9 & 7.5 & 6.5 & 0.5 & 13.4 & 13.9 & 13.9 \\
\hline 8.01-9.0 & 0.5 & 8.4 & 1.9 & 1.5 & 0.0 & 5.5 & 5.5 & 3.5 \\
\hline$>9$ & 3.4 & 5.9 & 2.9 & 1.9 & 0.0 & 4.5 & 4.5 & 4.9 \\
\hline
\end{tabular}

* $\mathrm{p}<0.05$

Porcentaje de pacientes en cada categoría de HbA1c, según tiempo de evolución de la enfermedad, manejo farmacológico para diabetes y adherencia al manejo.

${ }^{*} \mathrm{chi}^{2}$.

GLP1: péptido similar al glucagón tipo 1; HbA1c: hemoglobina glucosilada.

\section{DISCUSIÓN}

La DM es una de las patologías mas prevalentes a nivel mundial y produce una alta morbimortalidad, lo que puede mejorar con un buen control glucémico. Este estudio se realizó en la Fundación Valle del Lili, centro hospitalario donde se tratan todos los niveles de complejidad de la enfermedad y donde, además, se cuenta con representatividad de la mayoría de la población según estrato socioeconómico, afiliación al sistema seguridad social y nivel académico.

La prevalencia de la adherencia al tratamiento farmacológico en nuestra población (47.8\%) fue menor a la informada en estudios previos realizados en Bogotá y Cartagena (79.3 y $73 \%)^{9,10}$, pero con diferencias en el método utilizado para medirla, ya que en los estudios descritos evaluaron la adherencia general, no solo la farmacológica, como era el objetivo de nuestro estudio; otra diferencia es que utilizaban escalas análogas para su evaluación. A nivel mundial, los resultados al respecto difieren respecto a la adherencia farmacológica. En EE.UU. se ha reportado un $78,7 \%^{11}$; en Reino Unido ha variado desde $34^{12}$ a $87-89 \%^{13}$, si se calcula según reclamaciones administrativas o bases de datos, respectivamente. Como se observa, el rango es amplio y varía según los métodos utilizados para la medición, pudiendo sobrestimarse cuando se utilizan encuestas autoadministradas, como la utilizada en el presente estudio.

Hay múltiples factores que pueden estar relacionados con la adherencia y los resultados difieren según la población estudiada. Se ha hallado asociación con la edad, el sexo, el tipo de tratamiento, la comorbilidad y la polifarmacia ${ }^{14-17}$, todos con resultados contradictorios. En nuestro estudio, las únicas variables que se asociaron significativamente con la adherencia fueron el nivel académico y la procedencia: mayor nivel académico y proceder del área urbana resultaron factores protectores, similar a lo documentado en un estudio en EE.UU. ${ }^{16}$.

En cuanto al control glucémico, se encontró adecuado en el $53.7 \%$ de los casos, cifra mayor a la observada en el estudio realizado en Bogotá9. Las variables relacionadas fueron el tiempo de evolución de la enfermedad y el tratamiento para la misma, encontrando que, a mayor tiempo de evolución, mayor posibilidad de no estar controlado, esto probablemente con relación al curso de la enfermedad. También se encontró que la polimedicación no implica mayor control. 
No se documentó relación significativa entre adherencia y control glucémico, nuestro principal objetivo. Al respecto, numerosos estudios han encontrado una relación directa entre estas dos variables $^{10,11,18,19}$; no obstante, la variabilidad en la forma de medir adherencia hace difícil extraer una conclusión unificada al respecto.

Los resultados de nuestro estudio aportan una visión de los factores asociados al control glucémico y la adherencia al tratamiento farmacológico de la diabetes, sin documentar una relación directa entre ellos, lo que sugiere que hay otros factores implicados en el control más que el cumplimiento del tratamiento farmacológico propuesto. Nuestro estudio tiene limitaciones. La primera es que se utilizó un cuestionario autoadministrado para la valoración de la adherencia, lo cual puede incurrir en su sobrestimación; sin embargo, se empleó porque se encuentra validada para enfermedades crónicas como la DM, es fácil, corta y tiene alta especificidad y valor predictivo positivo. Otra limitación fue no contar con población del régimen subsidiado de salud. Con este estudio se plantea la necesidad de evaluar otros métodos para determinar la adherencia que puedan predecir de manera más eficaz el control glucémico, así como identificar otras variables vinculadas con este.

\section{BIBLIOGRAFÍA}

1. Forouhi NG, Wareham NJ. Epidemiology of diabetes. Medicine (Abingdon). 2014;42(12):698-702.

2. Aschner $\mathrm{P}$, King $\mathrm{H}$, Triana de Torrado $\mathrm{M}$, et al. Glucose intolerance in Colombia. A population-based survey in an urban community. Diabetes Care. 1993;16(1):90-3.
3. Lotufo PA, Gaziano JM, Chae CU, et al. Diabetes and all-cause and coronary heart disease mortality among US male physicians. Arch Intern Med. 2001;161(2):242-7.

4. Esmatjes E, Vidal J. [Heart pathology of extracardiac origin. XI. Cardiac repercussions of diabetes mellitus]. Rev Esp Cardiol. 1998;51(8):661-70.

5. Orchard TJ, Forrest KY, Ellis D, et al. Cumulative glycemic exposure and microvascular complications in insulin-dependent diabetes mellitus. The glycemic threshold revisited. Arch Intern Med. 1997;157(16): 1851-6.

6. Nathan DM, Genuth S, Lachin J, et al. The effect of intensive treatment of diabetes on the development and progression of long-term complications in insulin-dependent diabetes mellitus. N Engl J Med. 1993;329(14):977-86.

7. Effect of intensive blood-glucose control with metformin on complications in overweight patients with type 2 diabetes (UKPDS 34). UK Prospective Diabetes Study (UKPDS) Group. Lancet. 1998;352(9131): 854-65.

8. Morisky DE, Green LW, Levine DM. Concurrent and predictive validity of a self-reported measure of medication adherence. Med Care. 1986; 24(1):67-74

9. Alba LH, Bastidas C, Vivas JM, et al. Prevalence of glycemic control and associated factors in type 2 diabetes mellitus patients at the Hospital Universitario de San Ignacio, Bogota-Colombia. Gac Med Mex. 2009;145(6):469-74.

10. Lían AH, Andrade Y, Hernández $O$, et al. Personas con diabetes mellitus tipo 2 y su capacidad de agencia de autocuidado, Cartagena. Av Enferm. 2012;30(2):39-46.

11. Ho PM, Rumsfeld JS, Masoudi FA, et al. Effect of medication non adherence on hospitalization and mortality among patients with diabetes mellitus. Arch Intern Med. 2006;166(17):1836-41.

12. Buysman EK, Liu F, Hammer M, et al. Impact of medication adherence and persistence on clinical and economic outcomes in patients with type 2 diabetes treated with liraglutide: a retrospective cohort study. Adv Ther. 2015;32(4):341-55.

13. Farmer $A J$, Rogers LR, Lonergan $M$, et al. Adherence to oral glucose-lowering therapies and associations with 1-year HbA1c: A retrospective cohort analysis in a large primary care database. Diabetes Care. 2016;39(2):258-63.

14. Curkendall SM, Thomas N, Bell KF, et al. Predictors of medication adherence in patients with type 2 diabetes mellitus. Curr Med Res Opin. 2013;29(10):1275-86.

15. Bonafede MM, Kalsekar A, Pawaskar M, et al. Insulin use and persistence in patients with type 2 diabetes adding mealtime insulin to a basal regimen: a retrospective database analysis. BMC Endocr Disord. 2011;11:3.

16. Cramer JA. A systematic review of adherence with medications for diabetes. Diabetes Care. 2004;27(5):1218-24.

17. Jin J, Sklar GE, Min Sen Oh V, et al. Factors affecting therapeutic compliance: A review from the patient's perspective. Ther Clin Risk Manag. 2008;4(1):269-86.

18. Nichols GA, Rosales AG, Kimes TM, et al. Impact on glycated haemoglobin of a biological response-based measure of medication adherence. Diabetes Obes Metab. 2015;17(9):843-8.

19. Al-Qazaz H, Sulaiman SA, Hassali MA, et al. Diabetes knowledge, medication adherence and glycemic control among patients with type 2 diabetes. Int J Clin Pharm. 2011;33(6):1028-35. 